\title{
Constrained Wyner-Ziv Source Coding
}

\author{
Amos Lapidoth \\ ETH Zurich \\ CH-8092 Zurich, Switzerland \\ lapidoth@isi.ee.ethz.ch
}

\author{
Andreas Malär \\ ETH Zurich \\ CH-8092 Zurich, Switzerland \\ amalaer@ee.ethz.ch
}

\author{
Michèle Wigger \\ Telecom ParisTech \\ 75013 Paris, France \\ michele.wigger@telecom-paristech.fr
}

\begin{abstract}
We generalize the Wyner-Ziv source coding problem with side-information at the decoder to the case where the encoder is required to be able to compute the decoder's reconstruction sequence with some fidelity. This requirement limits the extent to which the reconstruction sequence can depend on the side-information, which is not available to the encoder.

For finite-alphabet memoryless sources and single-letter distortion measures we compute the minimal description rate as a function of the joint law of the source and side-information and of the allowed distortions at the encoder and decoder. We also treat memoryless Gaussian sources with mean squared-error distortion measures.
\end{abstract}

\section{INTRODUCTION AND SETUP}

We study an extension of the Wyner-Ziv source coding problem where the encoder is required to reproduce the decoder's reconstruction with some prespecified precision. This additional requirement limits the extent to which the decoder's reconstruction can depend on the side-information, which is not available to the encoder.

Our setting is specified by a tuple

$$
\left(\mathcal{X}, \mathcal{Y}, \hat{\mathcal{X}}, P_{X Y}, d_{\mathrm{d}}, d_{\mathrm{e}}, D_{\mathrm{d}}, D_{\mathrm{e}}\right),
$$

which we explain next. The set $\mathcal{X}$ is the source alphabet, the set $\mathcal{Y}$ is the side-information alphabet, and the set $\hat{\mathcal{X}}$ is the reconstruction alphabet. All are assumed finite except in our treatment of Gaussian sources where they are equal to the reals. The source and side-information sequence $\left\{\left(X_{i}, Y_{i}\right)\right\}_{i=1}^{n}$ is assumed to be drawn IID according to some joint law $P_{X Y}$ on $\mathcal{X} \times \mathcal{Y}$. The source sequence $X^{n}=\left(X_{1}, \ldots, X_{n}\right)$ is observed only at the encoder, and the side-information $Y^{n}=\left(Y_{1}, \ldots, Y_{n}\right)$ only at the decoder. We also specify two single-letter distortion functions $d_{\mathrm{d}}: \mathcal{X} \times \hat{\mathcal{X}} \rightarrow \mathbb{R}_{0}^{+}$and $d_{\mathrm{e}}: \hat{\mathcal{X}} \times \hat{\mathcal{X}} \rightarrow \mathbb{R}_{0}^{+}$. The former is used to measure the fidelity of the reconstruction at the decoder, and the latter to measure the fidelity with which the encoder estimates the decoder's reconstruction sequence. The allowed distortions are $D_{\mathrm{d}} \geq 0$ and $D_{\mathrm{e}} \geq 0$.

To describe the source sequence $X^{n}$, the encoder produces the index

$$
M=f^{(n)}\left(X^{n}\right)
$$

where $f^{(n)}: \mathcal{X}^{n} \rightarrow \mathcal{M}$ is the encoding function and $\mathcal{M}=$ $\{1, \ldots, \mathrm{M}\}$. The index $M$ is conveyed to the decoder who uses it and the side-information $Y^{n}$ to form the decoder's reconstruction sequence

$$
\hat{X}_{\mathrm{d}}^{n}=\phi^{(n)}\left(M, Y^{n}\right)
$$

where $\phi^{(n)}: \mathcal{M} \times \mathcal{Y}^{n} \rightarrow \hat{\mathcal{X}}^{n}$ is the decoder's reconstruction function. The encoder's estimate of the decoder's reconstruction sequence is

$$
\hat{X}_{e}^{n}=\psi^{(n)}\left(X^{n}\right)
$$

for some $\psi^{(n)}: \mathcal{X}^{n} \rightarrow \hat{\mathcal{X}}^{n}$.

We call a triplet of functions $\left(f^{(n)}, \phi^{(n)}, \psi^{(n)}\right)$ as above an $\left(n, R, D_{\mathrm{d}}, D_{\mathrm{e}}\right)$-code if $\mathrm{M} \leq 2^{n R}$ and the produced sequences $\hat{X}_{\mathrm{d}}^{n}=\left(\hat{X}_{\mathrm{d}, 1}, \ldots, \hat{X}_{\mathrm{d}, n}\right)$ and $\hat{X}_{\mathrm{e}}^{n}=\left(\hat{X}_{\mathrm{e}, 1}, \ldots, \hat{X}_{\mathrm{e}, n}\right)$ satisfy:

$$
\begin{gathered}
\frac{1}{n} \sum_{i=1}^{n} \mathrm{E}\left[d_{\mathrm{d}}\left(X_{i}, \hat{X}_{\mathrm{d}, i}\right)\right] \leq D_{\mathrm{d}} \\
\frac{1}{n} \sum_{i=1}^{n} \mathrm{E}\left[d_{\mathrm{e}}\left(\hat{X}_{\mathrm{d}, i}, \hat{X}_{\mathrm{e}, i}\right)\right] \leq D_{\mathrm{e}} .
\end{gathered}
$$

The nonnegative triplet $\left(R, D_{\mathrm{d}}, D_{\mathrm{e}}\right)$ is achievable if for every $\epsilon>0$ and sufficiently large $n$ there exists an $\left(n, R+\epsilon, D_{\mathrm{d}}+\right.$ $\left.\epsilon, D_{\mathrm{e}}+\epsilon\right)$-code. For a nonnegative distortion pair $\left(D_{\mathrm{d}}, D_{\mathrm{e}}\right)$ the rate-distortions function $R\left(D_{\mathrm{d}}, D_{\mathrm{e}}\right)$ is the infimum over all rates $R$ such that the triplet $\left(R, D_{\mathrm{d}}, D_{\mathrm{e}}\right)$ is achievable.

Our setup differs from the Wyner-Ziv setup [2] only in the additional constraint (4).

\section{RESULTS}

Theorem 1: If $\mathcal{X}, \mathcal{Y}, \hat{\mathcal{X}}$ are finite, then

$$
R\left(D_{\mathrm{d}}, D_{\mathrm{e}}\right)=\min _{Z, \phi, \Psi}(I(X ; Z)-I(Y ; Z))
$$

where $(X, Y) \sim P_{X Y}$, and where the minimization is over a discrete random variable $Z$ taking value in an auxiliary alphabet $\mathcal{Z}$ of size at most $|\mathcal{X}|+3$ and forming the Markov chain $Z \multimap X \multimap-Y$ and over the functions $\phi: \mathcal{Y} \times \mathcal{Z} \rightarrow \hat{\mathcal{X}}$ and $\psi: \mathcal{X} \times \mathcal{Z} \rightarrow \hat{\mathcal{X}}$ satisfying

$$
\begin{aligned}
\mathrm{E}\left[d_{\mathrm{d}}(X, \phi(Y, Z))\right] & \leq D_{\mathrm{d}} \\
\mathrm{E}\left[d_{\mathrm{e}}(\phi(Y, Z), \psi(X, Z))\right] & \leq D_{\mathrm{e}} .
\end{aligned}
$$

Remark 1: Let $I\{\cdot\}$ denote the indicator function. It can be shown that if

$$
d_{\mathrm{e}}\left(\hat{x}_{\mathrm{d}}, \hat{x}_{\mathrm{e}}\right)=I\left\{\hat{x}_{\mathrm{d}} \neq \hat{x}_{\mathrm{e}}\right\},
$$

then for all $D_{\mathrm{d}} \geq 0$ the expression for $R\left(D_{\mathrm{d}}, 0\right)$ in (5) coincides with Steinberg's common-reconstruction rate-distortion function $R_{\mathrm{cr}}\left(D_{\mathrm{d}}\right)$ in [1].

Theorem 2: Let $\mathcal{X}, \mathcal{Y}$, and $\hat{\mathcal{X}}$ be $\mathbb{R}$; let $P_{X Y}$ be the law of $(X, Y)$ where $X \sim \mathcal{N}\left(0, \sigma_{X}^{2}\right)$ and $Y=X+U$ for $U \sim$ 
$\mathcal{N}\left(0, \sigma_{U}^{2}\right)$ independent of $X$; and let $d_{\mathrm{d}}\left(x, \hat{x}_{\mathrm{d}}\right)=\left(x-\hat{x}_{\mathrm{d}}\right)^{2}$ and $d_{\mathrm{e}}\left(\hat{x}_{\mathrm{d}}, \hat{x}_{\mathrm{e}}\right)=\left(\hat{x}_{\mathrm{d}}-\hat{x}_{\mathrm{e}}\right)^{2}$.

- If $\sqrt{D_{\mathrm{e}} \sigma_{U}^{2}} \geq \min \left\{D_{\mathrm{d}}, \frac{\sigma_{X}^{2} \sigma_{U}^{2}}{\sigma_{X}^{2}+\sigma_{U}^{2}}\right\}$, then

$$
R\left(D_{\mathrm{d}}, D_{\mathrm{e}}\right)=\max \left\{0, \frac{1}{2} \log \left(\frac{\sigma_{X}^{2} \sigma_{U}^{2}}{\left(\sigma_{X}^{2}+\sigma_{U}^{2}\right) D_{\mathrm{d}}}\right)\right\} \text {. }
$$

- If $\sqrt{D_{\mathrm{e}} \sigma_{U}^{2}}<\min \left\{D_{\mathrm{d}}, \frac{\sigma_{X}^{2} \sigma_{U}^{2}}{\sigma_{X}^{2}+\sigma_{U}^{2}}\right\}$, then

$$
\begin{aligned}
& R\left(D_{\mathrm{d}}, D_{\mathrm{e}}\right) \\
& =\max \left\{0, \frac{1}{2} \log \left(\frac{\sigma_{X}^{2}}{\sigma_{X}^{2}+\sigma_{U}^{2}} \frac{\sigma_{U}^{2}+D_{\mathrm{d}}-2 \sqrt{\sigma_{U}^{2} D_{\mathrm{e}}}}{D_{\mathrm{d}}-D_{\mathrm{e}}}\right)\right\} .
\end{aligned}
$$

Remark 2: If

$$
\sqrt{D_{\mathrm{e}} \sigma_{U}^{2}} \geq \min \left\{D_{\mathrm{d}}, \frac{\sigma_{X}^{2} \sigma_{U}^{2}}{\sigma_{X}^{2}+\sigma_{U}^{2}}\right\}
$$

or

$$
\left(1-\sqrt{\frac{D_{\mathrm{e}}}{\sigma_{U}^{2}}}\right)^{2} \sigma_{X}^{2} \leq D_{\mathrm{d}}-D_{\mathrm{e}}
$$

then our result coincides with Wyner and Ziv's result [2]. Thus, in this case removing constraint (4) or revealing the side-information also to the encoder do not decrease the ratedistortion function.

\section{REFERENCES}

[1] Y. Steinberg, "Coding and Common Reconstruction," IEEE Transactions on Information Theory, vol. 55, no. 11, pp. 4995-5010, Nov. 2009.

[2] A. D. Wyner and J. Ziv, "The Rate-Distortion Function for Source Coding with Side Information at the Decoder", IEEE Transactions on Information Theory, vol. 22, no. 1, Jan. 1976. 